\title{
Genesis of the planet Earth
}

\begin{abstract}
In this article we consider the origin and subsequent evolution of our planet. Theoretical studies are based on the author's theory of vortex gravity, cosmology and cosmogony, and also on the law of conservation of the angular momentum of the body's rotation. As a practical justification for calculations and conclusions, the well-known fact of slowing the rotation of the Earth is used. The principles of the formation of elementary particles and all types of inorganic and organic substances are proposed. The calculation of the physical state of our planet in the past and in the future is done. The age of the Earth is calculated in the article, which exceeds the generally accepted value by 1.2 billion years. It is shown that the principle of the origin and evolution of the Earth is fully applicable for the genesis of all other celestial bodies.
\end{abstract}

Keywords: theory of vortex gravitation, celestial mechanics, cosmology, cosmogony
Volume I Issue I - 2017

\section{Sergey Orlov}

Petrozavodsk State University, Petrozavodsk, Russia

Correspondence: Sergey Orlov, Petrozavodsk State University, Petrozavodsk, Russia, Email ion@sampo.ru

Received: June 14, 2017| Published: July 17, 2017

\section{Introduction}

From the earliest ages people believed that the Earth's rotation around its axis and revolves around the Sun has always happened and is happening always in identical periods. Doubts about the constancy of the speed of rotation of the earth arose after the discovery of $\mathrm{E}$ Halley in 1695 secular acceleration of the moon's motion. The idea of the secular slowing rotation of the Earth under the influence of tidal friction was first proposed by Kantin 1755 Nowadays Richard Stephenson from Durham University in the UK, based on the descriptions of hundreds of solar and lunar eclipses last 2,700years, came to the conclusion that the Earth continues slow. ${ }^{1}$ Cause slowing of the Earth is called by many factors. These include the gravitational influence of the moon and sun, braking cosmic dust, atmospheric and geophysical processes and many other physical phenomena. In this paper, conventional explanations irregular rotation of the earth understood as an unproven and (or) is negligibly small. External gravitational influences or atmospheric effects cannot only slow, but also accelerate the Earth's rotation. The genesis of our planet, including the slowing of its rotation, fully explained by the theory of vortex gravitation, cosmology and cosmogony ${ }^{2}$ and the laws of mechanics. For greater clarity, the readers, the next chapter outlines the principles of the theory of vortex gravitation.

\section{The theory of vortex gravitation}

Vortex gravitation theory ${ }^{2}$ is based on the well-known astronomical facts-all celestial objects revolve. The most logical explanation of the cause of this movement can be only one - the rotation of celestial objects spawned vortex rotation of cosmic matter-ether. Ether forms a global system of interconnected space vortices. Orbital velocity in each vortex ether (torsion), decreasing from the center to the periphery of the law of the inverse square of the removal. According to the laws of aerodynamics-the slower the rate, the more pressure there. The pressure gradient gene rates a force pushing towards the zone with the lowest pressure to the center of the torsion bar (Figure 1). Thus accumulates in the center of the torsion or created cosmic matter of which is generated by celestial body. Consider the equation of vortex gravitation theory obtained in the theory of vortex gravitation, cosmology and cosmogony. Shown forces acting on the body 2. $\mathrm{F}_{\mathrm{c}}$-centrifugal force $\mathrm{F}_{\mathrm{n}}$-attraction force of body 2 to body 1 ,
$\mathrm{V}_{2}$-linear velocity of a body in orbit $2, \mathrm{R}$ - radius of the orbit, $\mathrm{r}_{1}$ radius of the body $1, \mathrm{r}_{2}$-radius of the body $2, \mathrm{w}_{1}$-the angular velocity of rotation of the ether on the surface of the body $1, \mathrm{~m}_{2}-$ mass of the body 2 . As already mentioned, the result of motion of the vortex pressure gradient arises. Radial distribution of pressure and velocity in the ether ${ }^{2}$ defined on the basis of the Navier-Stokes equations for the motion of a viscous fluid (gas).

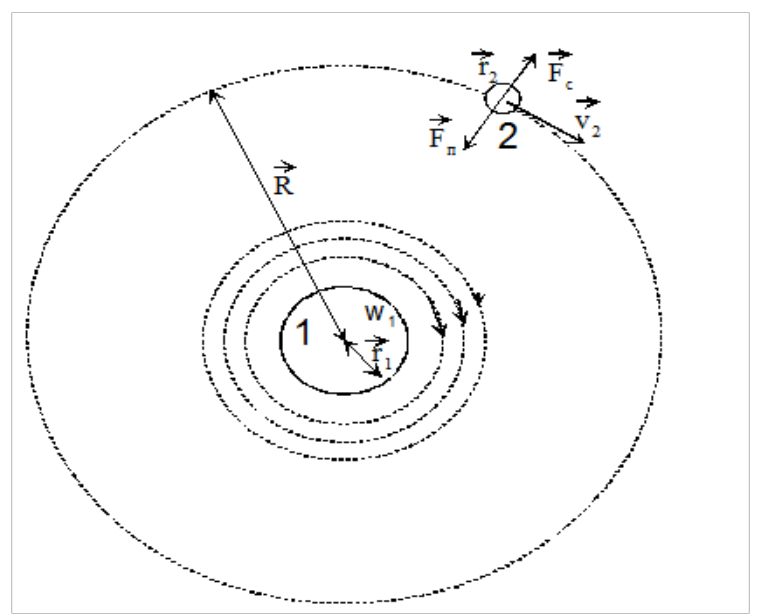

Figure I Two-dimensional model of the gravitational interaction between the two bodies.

$$
\rho\left[\frac{\partial}{\partial t}+\vec{v} \cdot \operatorname{grad}\right] \vec{v}=\vec{F}-\operatorname{grad} \mathrm{P}+\eta \Delta \vec{v}
$$

In cylindrical coordinates, taking into account the radial symmetry $\mathrm{v}_{\mathrm{r}}=\mathrm{v}_{\mathrm{z}}=0=\mathrm{v}(\mathrm{r}), \mathrm{P}=\mathrm{P}(\mathrm{r}) \phi$, equation can be written as a system.

$$
\left\{\begin{array}{l}
-\frac{v(r)^{2}}{r}=-\frac{1}{\rho} \frac{d P}{d r} \\
\eta \cdot\left(\frac{\partial^{2} v(r)}{\partial r^{2}}+\frac{\partial v(r)}{r \partial r}-\frac{v(r)}{r^{2}}\right)=0
\end{array}\right.
$$

Where $\rho=8.85 \times 10^{-12} \mathrm{~kg} / \mathrm{m}^{3}$ the density of, ${ }^{4}$ V-ether velocity vector, $\mathrm{P}$ - pressure ether, $\eta$-viscosity. 
In cylindrical coordinates module gravity

$$
F_{\mathrm{i}}=V \cdot \frac{\partial \mathrm{P}}{\partial r}
$$

Then comparing (2) and (3) for an in compressible there $(\rho=$ const), we find that

$$
R_{-1}=6,371 \times 10^{6} \times 0,94=5,988 \times 10^{6} \mathbf{i}
$$

The assumption № 1 - Ether pervades all space, including the physical body except nucleons. Volume V in the formula (4) an effective volume the volume of the elementary particles that make up the body 2. All bodies are composed of electrons, protons and neutrons. Radius of the electron is much smaller than the radius of the proton and the neutron radius is approximately the same and the last of the order $r_{n} \sim 1.2 \times 10^{-15} \mathrm{~m}$ masses of the proton and neutron are approximately the same as $m_{n} \sim 1.67 \times 10^{-27} \mathrm{~kg}$ ( $\mathrm{rn}, \mathrm{mn}-$ the radius and mass of the nucleon). After the necessary transformation (full payment is set out in the theory vortex gravitation) is obtained:

1) Equation for determining the force of gravity depending on the rotational speed ether

$$
F_{\mathrm{i}}=\frac{4 \cdot \pi \cdot r_{n}^{3} \cdot \rho}{3 \cdot m_{n}} \cdot \frac{w_{1}^{2} \cdot r_{1}^{3} \cdot m_{2}}{r^{2}}
$$

$r_{n}, m_{n}$-the radius and mass of the nucleon.

2) Equations for determining the dependence of the pressure on the body surface $\mathrm{P}_{0}$, the rotational speed ether

$$
\mathrm{P}_{0}=\mathrm{P}_{b}-\rho \cdot w_{1}^{2} \cdot r_{1}^{2}
$$

Where $\mathrm{P}_{0}$ - ether pressure at the surface of the body, using the boundary condition $\mathrm{P}_{\mathrm{b}}$-free ether pressure. Figure 2 is a graph showing the pressure distribution in accordance with formula (6).

\section{Changes in volume and mass of the earth}

According to the theory of vortex gravitation, cosmology and cosmogony, the initial moment of the appearance of any celestial body was the emergence of space, ether vortex. At the time of its inception, each vortex created his vortex gravity (Chapter 2). Vortex gravity can be regarded as "generator world of matter " that sucks and (or) creates a vortex inside the elementary particles. Thus, the mass of each celestial object in the initial moment of its existence was equal to the mass of the cosmic ether, from which it was formed vortex. Since the density of the ether is negligible, then the mass of each of the newly formed vortex ether should be close to zero. Consequently, each celestial body in the history of its existence increased its weight from "zero conditional" to these values. It is worth noting an important condition - cosmic whirlwind unopposed, constantly maintained and retains its original speed (see assumption. № 1). Therefore, the space whirlwind constantly generates the same mass of substance. That is any celestial body increases the weight by a constant. Modern studies customary are slowing the Earth's rotation value 0.00002 seconds in each year. ${ }^{1}$ Then the relative increase in time for the Earth's rotation around its axis is increased by a factor $\mathrm{K}_{\mathrm{t}}$.

$\mathrm{K}_{t}=(24 \times 60 \times 60+0,00002) \times(24 \times 60 \times 60)^{-1}=1+2,314 \times 10^{-10}$

By increasing the time for one revolution of the earth is inversely proportional to the rotational speed decreases $\mathrm{V}_{\mathrm{e}}$. Then the relative decrease in the rate of rotation of $\mathrm{K}_{\mathrm{v}}$ can be expressed as

$$
\hat{\mathbf{E}}_{v}=\mathrm{K}_{t}^{-1}=\left(1+2,314 \times 10^{-10}\right)^{-1}
$$

Further calculations are based on the law of conservation of angular momentum the Earth's rotation around its axis.

$$
M V_{v} R_{e}=\text { const }
$$

$\mathrm{M}=$ mass of the planet, $\mathrm{V}_{\mathrm{v}}=$ the speed of rotation of the planet and $\mathrm{R}_{\mathrm{e}}=$ the radius of the planet. From equation (8)

$$
M V_{\mathbf{v}} \mathbf{R}_{\mathrm{e}}=\left(\mathbf{K}_{\mathbf{m}} \mathbf{M}\right)\left(\mathbf{K}_{\mathbf{v v}} \mathbf{V}_{\mathbf{v}}\right)\left(\mathbf{K r}_{\mathbf{e}} \mathbf{R}_{\mathbf{e}}\right)=\text { const }
$$

Where the coefficients $\mathrm{K}_{\mathrm{m}}, \mathrm{K}_{\mathrm{vv}}, \mathrm{K}_{\mathrm{re}}$ are show the relative changes in the valuesM, $V_{v}, R_{e}$. Hence

$$
\mathbf{K}_{\mathbf{m}} \mathbf{K v} \mathbf{K r}_{\mathbf{e}}=\mathbf{1}
$$

Substituting (7) into (9) we obtain

$$
\mathrm{K}_{m} \mathrm{~K}_{r e}=1+2,314 \times 10^{-10}
$$

Planet mass(M),as well asits volume(V)is proportional to the radius of the planet in the cube.

$$
\begin{aligned}
& \mathbf{M} \sim \mathbf{V} \sim \mathbf{R e}^{3} \text { here } \\
& \mathbf{K r}_{\mathbf{e}}{ }^{3}=\mathbf{K}_{\mathbf{m}}
\end{aligned}
$$

Substituting (11) into (10)

$$
\begin{gathered}
\mathrm{K}_{r}{ }^{4}=1+2,314 \times 10^{-10} \text { or } \\
\mathrm{K}_{r}=\left(1+2,314 \times 10^{-10}\right)^{1 / 4}=1+5,785 \times 10^{-11}
\end{gathered}
$$

Substituting (12) into (10) we determine the relative increase in the mass of the Earth-

$$
\mathrm{K}_{m}=1+1,735 \times 10^{-10}
$$

\section{To determine the absolute values of the physical characteristics of the earth}

I. At rotation speed of the Earth surface at the equator $V_{v}=465,1 \mathrm{~m} / \mathrm{s}$, annual slowdown will

$$
465,1 \times 2,314 \times 10^{-10}=9,3 \times 10^{-8} \mathbf{i} / \text { seK }
$$

II. When the radius of the Earth $R_{e}=6371000$ manual increase in the radius of the Earth

$$
6371000 \times 5,785 \times 10^{-11}=3,7 \times 10^{-4} \grave{\mathbf{i}} \text { èëè } 0,37 \mathbf{i} \mathbf{i ̀ ~} / \text { year }
$$

Or $37 \mathrm{~cm}$ in 1,000 years or $370 \mathrm{~m} 1000$ 000years

Therefore, the earth deposits at a depth of about $370 \mathrm{~m}$ have and average age of the sediments million years ago.

III. When the mass of the Earth $M=5.9736 \times 10^{24} \mathrm{~kg}$ annual weight gain

$$
5,9736 \times 10^{24} \times 1.735 \times 10^{-10}=1,036 \times 10^{15} \kappa g
$$

Increased Earth The surface area of the Earth $S_{v}=4 M R^{2}$.Increasing the radius of $-3.7 \times 10^{-4} \mathrm{~m}$ then the volume of the Earth increases by

$$
4 \ddot{\mathrm{I}} R^{2} \times 3,7 \times 10^{-4}=4 \tilde{\mathbf{o}} 3.14 \times\left(6,371 \times 10^{6}\right)^{2} \times 3,7 \times 10^{-4}=1,886 \times 10^{11}{ }^{3}
$$

IV. Additional mass density

$$
\mathrm{P}=3,456 \times 10^{14} \times\left(1,835 \times 10^{11}\right)^{-1}=1883 \kappa g / \mathbf{i}^{3}
$$


Note: The absolute value of the above characteristics of the Earth is only valid in the present historical moment, as the speed, radius and mass of the planet are constantly changing.

\section{Mass and age of the earth, orbital and radial motion}

Earth's mass is constantly increasing the amount of (13)

$$
\mathbf{K}_{\mathbf{m}}=1.735 \times 10^{-10} x \mathbf{M}
$$

No outside forces act on the planet. Therefore, legitimate to use the law of conservation of angular momentum of the Earth around the Sun.

$$
\begin{aligned}
& M V_{0} R_{0}=\text { const, where } \\
& \mathbf{K}_{\mathbf{m}} \mathbf{K}_{\mathrm{vo}} \mathbf{K}_{\text {ro }}=\mathbf{1}
\end{aligned}
$$

$\mathrm{M}$-mass of the Earth

$\mathrm{V}_{\mathrm{o}}$-the orbital speed of the Earth

$\mathrm{R}_{\mathrm{o}}$-radius of Earth's orbit.

$\mathrm{K}_{\mathrm{m}}, \mathrm{K}_{\mathrm{vo}}, \mathrm{K}_{\mathrm{ro}}$ - rate of change in the Earth's mass units, the speed of its revolution around the Sun and the orbit radius.

According to Kepler's law $V \sim R_{o}^{-1 / 2}$, we can write

$$
\mathrm{K}_{\mathrm{vo}}=\mathrm{K}_{\mathrm{r}}^{-1 / 2}
$$

Substitute (20) into (19)

$$
\mathrm{K}_{r o}=\mathrm{K}_{m}{ }^{-2}=\left(1+1.735 \times 10^{-10}\right)^{-2}=\left(1+3,47 \times 10^{-10}\right)^{-1}
$$

Substituting (21) and (13) into equation (19) we obtain

$\mathrm{K} v_{o}=\left(\mathrm{K} m x \mathrm{~K} r_{o}\right)^{-1}=\left(1+3,47 \times 10^{-10}\right) \times\left(1+1.735 \times 10^{-10}\right)^{-1}=1+1.735 \times 10^{-10}$

Mass of the planet increases always constant. Therefore, by dividing the mass of the Earth on its permanent annual increase $\left(\mathrm{M} \times 1.735 \times 10^{-10}\right)$, we obtain the age of the planet

$$
\mathrm{T}=\mathrm{M} \times(\mathrm{M} \times \mathrm{Km})^{-1}=\mathrm{M} \times\left(\mathrm{M} \times 1.735 \times 10^{-10}\right)^{-1}=\left(1,735 \times 10^{-10}\right)^{-1}=5,76
$$

billion years Radio isotope dating ${ }^{3}$ established the age of the planet size of 4.54 billion years. It should be noted that the radioisotope dating explored only the surface layers of the planet. Therefore, the results of these studies (age) can be attributed only to the same surface layers. The proposed method of determining the age of the planet considering as a single physical object, which increases the reliability of its results. Annual approaching the Earth's orbit to the Sun

$R_{o} \times \mathrm{K}_{r o}=15 \tilde{\mathbf{o}} 10^{10} \times 3,47 \times 10^{-10}=52,05 \mathrm{~m} /$ year And One revolution of the Earth around the sun(one year)is reduced by: $365,24($ day $) \times 24 \times 60 \times 60 \times 1,735 \times 10^{-10}=0,0054 \mathrm{sec} /$ year

\section{Past and the future of the planet}

Calculation of physical properties in the past and in the future.

\section{000000000 years ago}

\section{a) The rotation of the planet}

Mass of the planet billion years ago

$$
1,036 \times 10^{15}(\mathrm{~kg}) \times\left(4.76 \times 10^{9}\right)(\mathrm{s})=4.93 \times 10^{24} \mathrm{~kg}
$$

Mass ratio $K_{m-1}=4.93 \times 1024 / 5,97 \times 1024=0,826$

The relative change Earth radius

$$
K_{r-1}=\left(K_{m-1}\right)^{1 / 3}=(o, 826)^{1 / 3}=0.94
$$

From equation (19),

$$
K_{v-1}=\left(K_{r} x K_{m-1}\right)^{-1}=\left(\begin{array}{lll}
0.826 x & 0.94
\end{array}\right)^{-1}=1.288
$$

Absolute values of the planet a billion years ago. Since the velocity of the planet was more than1,288 times, in Length of day a billion years ago-18.6hours Radius of the planet was less than 0.94 times or

$\mathbf{K}_{\mathbf{r} 0-1}=1.466$. Consequently, the force of gravity was greater in $(0.94)^{-1 / 2}=(\mathbf{0 . 8 8})^{-1}$ times. That is $m_{n} \sim 1.67 \times 10^{-27} \mathrm{~kg}$

\section{Handling the planet}

Using formulas 20, 21,22 and $\mathbf{K}_{\mathbf{m}-\mathbf{1}}=\mathbf{0 . 8 2 6}$, we find:

- The ratio of the radii of $\mathrm{K}_{r 0-1}=1.466$

-The relative decrease in orbital velocity (1billion years ago) -

$\mathrm{K}_{\text {vi }-1}=0,826$

-The orbital speed $V_{0-1}=29,8 \times 10^{3} \times 0,826=24,6 \times 10^{3} \mathrm{~m} / \mathrm{s}$

- The ratio of the radius

$$
R_{o-1}=R \times \mathrm{K}_{r o-1}=15 \times 10^{10} \times 1,466=219,9 \mathrm{~m} \ln \kappa m
$$
days.

The number of days (18.6 hours).Per revolution (one year)-838,4

\section{After I 000000000 years in the future}

\section{a) The rotation of the planet}

Mass of the planet a billion years

$$
1,036 \times 10^{15}(\mathrm{~kg}) \times 6,76 \times 10^{9} \text { (years) }=7,0 \times 10^{24} \mathrm{\kappa g}
$$

Changing the masses $\mathrm{K}_{m+1}=7,0 \times 10^{24} / 5,97 \times 10^{24}=1,173$

The relative change radius of the Earth

$$
\mathrm{K}_{r+1}=\left(\mathrm{K}_{m+1}\right)^{1 / 3}=(1,173)^{1 / 3}=1,055
$$

From equation (19),

$$
\mathrm{K}_{v+1}=\left(\mathrm{K}_{r+1} \times \mathrm{K}_{m+1}\right)^{-1}=(1,055 \tilde{\mathbf{o}} 1,173)^{-1}=0,81
$$

The absolute values of the properties of the planet in the future in a billion years.

Since the rotation speed of the planets will be less than 0.81 times, then

Length of the day 29.6hours

Radius of the planet $R_{+1}=6,721 \times 10^{6} \mathrm{~m}$

The gravitational force $F=8,8 \mathrm{~m}$

\section{b) Handling the planet}

Using formulas $20,21,22$ and $\mathrm{K}_{m+1}=1,173$,we find: future)

The relative increase in orbital velocity(in billion years in the 


$$
\mathrm{K}_{v \hat{\mathbf{1}}+1}=1,042
$$

Relation of orbital radiuses $\mathrm{K}_{r o+1}=0,727$

Radius of the orbit

$$
R_{v 0+1}=R \times K_{r 0+1}=15 \times 10^{10} \times 0,727=109 \mathrm{mln} \mathbf{~ k m}
$$

The orbital speed of $V_{0}+1=29.8 \times 103 \times 1,042 \times 103=31.05 \mathrm{~m} / \mathrm{s}$

Number of days (29.6 hours) per revolution (year) -206.9

Note 1: The proposed calculations of the radius of Earth's orbit are valid only if the orbital plane is considered a celestial body (the Earth) coincides with the center plane of the gravitational ether vortex. In the event of the orbital plane of the celestial body of the plane gravitational torsion vortex gravitation force is reduced in proportion to the cube of the cosine of the deviation. A detailed calculation of this dependence is presented in the theory of vortex gravitation. Orbital plane of the Earth's gravity deviates from the plane by degree. Consequently, the tops of the small semi axes earth orbit force swirl solar gravity decreases by $0.045 \%$ in comparison with the magnitude of gravity, which was determined by the classical equations. Therefore, changing the radius of the orbit of the Earth or the duration of the year in the past or the future, calculated in this article actually has smaller values. For large deviations of the orbital planes of any celestial object and the long duration of their existence can remove the orbital trajectories of these objects from the call center. Such removal ( $38 \mathrm{~mm}$ per year) is currently seen in the moon.

\section{Creation of substance of the planet}

Most modern scholars explain the increased mass of the planet and meteorite dust flux of cosmic matter on Earth. The magnitude of this cosmic matter is determined by researchers in the order of several tens of thousands of tons $\left(10^{7} \mathrm{~kg}\right)$ per year. In this paper we calculated that the observed slowing rotation of the planet can only be achieved by increasing the mass of the planet at $\mathbf{1 , 0 3 6} \times \mathbf{x 1 0}^{\mathbf{1 5}} \mathbf{~} \mathbf{k g}$ per year (the equation16). This calculated weight exceeds the estimated mass of cosmic matter that falls to Earth from space, hundreds of millions of times. Consequently, the total mass of cosmic dust and meteorites falling annually on our planet is negligible. Therefore, the study of increasing the mass of the Earth, the mass of meteorites can be neglected and consider another source of creation of matter. Based on the principles of vortex gravitation and cosmogony- the substances of all celestial bodies (elementary particles) create ethereal vortices.

Consider the principles of vortex cosmogony.

According to the theory of ether-dynamics ${ }^{4}$ the following parameters of the ether:- Pressure in the quiet, motionless air $2 \times 10^{32} \mathrm{~nm}^{-2}$, Ether density $\rho=8.85 \times 10^{-12} \mathbf{k g} / \mathrm{m}^{3}$

On the basis of equation (6) at a speed of ether $v_{0}=4,75 \times 10^{21}$ Earth torsion pressure should drop to zero (Figure 2$)^{0}$. We define the radius of the orbit of the Earth torsion with zero pressure.

On the basis of equation (5) determine the rotational velocity of the ether on the Earth's surface is $v_{e}=1.277 \times 10^{18} \mathrm{~m} / \mathrm{c}$ Transform equation (20) -

$$
\frac{V_{0}^{2}}{V_{e}^{2}}=\frac{r_{e}}{r_{0}}, \text { where }
$$

$v_{e}=1.277 \times 10^{18} \mathrm{~m} / \mathrm{c}$ - The orbital velocity of the ether on the surface of the Earth $v_{0}=4,75 \times 10^{21} \mathrm{~m} / \mathrm{c}$ - ether velocity in orbit with zero pressure

$r=6371000 m$-radius of the Earth and $r_{0}=$ earth orbit radius of the vortex, where the pressure is zero (no orbit). Substituting the known values of $\mathrm{v}_{0}, \mathrm{v}_{\mathrm{e}}, \mathrm{r}_{\mathrm{e}}$, define $\mathrm{r}_{0}$.

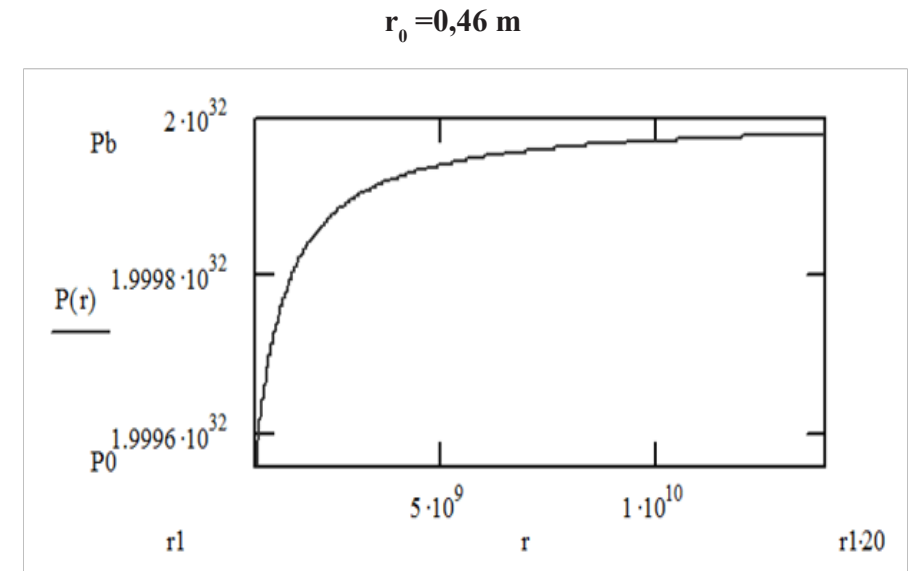

Figure 2 Radial distribution of ether pressure for the Sun.

Zero pressure in the Earth's orbit to any torsion means cessation of ether motion on this orbit, which caused reduction (increase) in ether pressure to the initial value in the quiescent state $2 \times 10^{32} \mathrm{Nm}^{-2}$. Note. Meteorologists note full tranquility (calm) in the center of tropical or sea storms. These phenomena are called "a storm eye". The sharp increase in pressure in the central orbits torsion in these areas will create a vortex antigravity force directed from the center of the torsion of the orbit with zero pressure $(46 \mathrm{~cm})$.

Thus, on the above, the zero orbit the Earth torsion, on both sides, there are two huge forces over the vortex gravitation. From equation (5) determine the gravitational force $\left(\mathrm{F}_{0}\right)$ in the Earth's orbit on the torsion bar with a radius of $0.46 \mathrm{~m} . \mathbf{F}_{\mathbf{0}}=\mathbf{1 , 9} \times \mathbf{\times 1 0}{ }^{15} \mathbf{m}$

Two opposite forces of gravity give rise to corresponding compression forces that cause the seal over rarefied ether to super dense state. Thus, in the center of any cosmic torsion created over dense core, which does not pass through it any radiation, including air. In the downtown core - the emptiness. At the same time, the core material is impermeable barrier to the orbital rotation of the ether on the orbits of the orbit below the outer surface of the core. When driving close to the surface of the ether in its core flow turbulence arises, twist and many micro vortices. These micro vortices similar planetary torsion, its own gravity pulls and seals the outer gaseous. In the center of micro-torsions particle density reaches the nucleon density $-10^{17} \mathrm{~kg} / \mathrm{m}^{3}$. It should be noted that the establishment of microtorsions occur at higher orbits the Earth torsion than the orbit of the nucleus. Vortex gravitation force decreases with increasing orbit. This allows not only nucleons, but also atoms. Continuously generated by the atoms of various substances in the central space of the vortex, generate celestial bodies. Throughout the history of the mega all the heavenly bodies the formation of atoms - continuous. Pouring into the magma, the newly formed atoms constantly "feed" the heavenly bodies more weight. Magma periodically overflowing through the Earth's crust cracks comes to the surface as lava. Geologists estimate the total mass of lava poured out a year tens of cubic kilometers, or $10^{12}$ cubic meters. The average population density in the order of lava $10^{3} \mathrm{~kg} / \mathrm{m}^{3}$, its mass is measured about $10^{15} \mathrm{~kg}$. Consequently, the actual 
mass of lava poured out annually, representing an increase of mass of the planet, according to the proposed settlement. This proves the accuracy of the proposed study on age and change in the physical parameters of the Earth.

The mere appearance of the substance (lava) from the depths of the planet proves that the substance appeared in the bowels of the earth, and was not delivered from space. This statement is obvious as meteorites or a space dust can't get in a planet body. Continuous creation of substance from ether in a planet possibly only at continuous inflow of ether to the planetary torsion. We will determine the volume of ether necessary for creation of $10^{15} \mathrm{~kg}$ of planetary substance. An increase in mass of Earth has to be equal to the mass of ether from which this additional mass of a planet was created. If we share quantity of annual increase in mass of Earth into ether density, we will determine the volume of ether which annually is required for substance weighing $10^{15}$ creation.

$$
V_{e}=m / r=10^{15} / 8.85 \times 10^{-12}=1,13 \times 10^{26} \mathrm{~m}^{3}
$$

This volume corresponds to the volume of the ether's torsion with a radius of 1 millionkm and with an axial thickness of 100 thousand $\mathrm{km}$. All of the above principles of vortex cosmogony apply to all celestial bodies in our universe. The main conclusion of Chapter 6 the substance is created by cosmic vortices of ether inside of celestial bodies. Moving the matter in the universe from one celestial body to another has only a minor character and the physical properties of celestial bodies affect. Generated in the Earth's core atoms of chemical elements are constantly overwhelmed this kernel and create a giant pressure overlying layers of the planet. These pressures exceed sin magnitude the opposite direction to him the gravitational pull of the Earth. Thus, the overlying layers of the earth continuously shifted by the lower layers towards the surface. During this movement, the chemical elements at certain depths enter the geophysical processes and form we all known inorganic compound-rock, hydrocarbons, water, and other gases. At a certain moment in history, these deposits are close to the earth's crust, or come to the surface of the Earth. Consequently all the necessary human civilization minerals-inexhaustible. In place of the explored and develop hydrocarbons, diamonds, gold and other precious natural resources for humans, the Earth will create new and thrust them out of their depth in the crust or on the surface. The proposed principles of inorganic matter, just as likely to apply to the creation of an organic substance. Only certain earth form actions are extreme chemical and physical conditions which permit the synthesis of organic molecules and their compounds. Obviously, on the surface of planets create conditions impossible. Consequently, there cannot be a synthesis of organic matter. Organic matter, since its inception in the depths of the heavenly bodies, in certain historical moments erupts on the surface of the Earth, together with inorganic substances.
For the development of the organic substance to a variety of animal and plant species on the Earth's surface requires the existence of favorable natural conditions, primarily these include optimal vortex gravitation force on the surface of the planet and the solar heat and light exposure. Not only plant and animal species emerged from the bowels of the planet, but also pathogenic viruses and bacteria, as evidenced by the annals of weather disasters and epidemics. Message from Thucydides (c.460-400BC) is known, that the epidemic that raged in Atticabetween 436 and 427 years. $\mathrm{BC}$, accompanied by strong earthquakes, sea floods, drought sand crop failures. In Europe, after the eruption of Etna in 1333, broke out "Black Death" - the bubonic and pneumonic plague, bevel most of the population of medieval Europe (about60 million). In article ${ }^{5} \mathrm{~A}$. Chizhevsky noted numerous cases of the relationship of animal diseases and plant protection and catastrophic atmospheric phenomena. Consequently, most commonly known to mankind world catastrophes (floods, glaciations, pandemics, tectonic movements of the crust, etc.), as well as the emergence and transformation of the atmosphere, hydrosphere, biosphere, topography and climate occurred and are occurring as a result of emissions to the surface of terrestrial matter. Meteorites and cosmic dust have on the development of the biosphere is only minor and insignificant impact. The basis of all organic and inorganic matter ether. For the development of air into living or non-living body, that is, for their origin and evolution are necessary extreme conditions that can only be created inside a celestial body and the corresponding vortex. Thus, space, ether vortices - creators of all known forms and types of matter.

\section{Acknowledgments}

None.

\section{Conflicts of interest}

The author declares there is no conflict of interest.

\section{References}

1. F Richard Stephenson. How Reliable are Archaic Records of Large Solar Eclipses? Journal for the History of Astronomy. 2008;39(4):229-250.

2. S Orlov. Foundation of vortex gravitation, cosmology and cosmogony. Global journal of science Frontier research. Physic and Space Science. 2012;12(1)

3. Dalrymple G Brent. The age of the Earth in the twentieth century: a problem (mostly) solved Special Publications. Geological Society of London. 2001;190(1):205-221.

4. VA Atsurovskiy. General ether-dynamics. Energoatomizdat. Moscow, Russia. 1990. p. 278.

5. AL Chizhevsky. Epidemic catastrophes and periodic activity of the Sun. 1930. p.172. 